\title{
JUSTICE IN THE COVID-19 PANDEMIC: MOBILITY CAPITAL AND NEW SOCIAL ORDER
}

\author{
Evgeniy V. Karchagin \\ Volgograd State Technical University, Volgograd, Russian Federation
}

\begin{abstract}
The COVID-19 pandemic forces us to reconsider the conceptual boundaries of the world and everyday social order, affecting such pairs of concepts as: natural / artificial; habitual / extraordinary. The author considers one of the aspects of the changes having occured: the transformation of spatial mobility, which is connected with deep social changes. In the first part, the experience of isolation is interpreted on the basis of the theoretical resources of the social theory of mobilities, primarily the concepts of mobility capital and mobility justice. Not all social groups were equally mobile, because they had different mobility capital. The issue of mobility equity has taken in a new context: a natural global threat that has exacerbated the existing inequalities caused by the emergency. The second part of the article deals with the concept of "state of emergency" by G. Agamben and analyzes the issue of transgression of the system of the world social order, including its everyday dimension. The answer to this question is given on the basis of an analysis of the interpretations and forecasts of the leading contemporary European intellectuals (Agamben, Žižek, Latour, Sloterdijk, Fuller). The problems of social distancing, the transformation of higher education, the increase in the powers of the state, associated with medical justifications are considered. Important parameters of the new social order are the environmental factor and the need for sociocritical optics to understand the consequences of the pandemic. Analysis captures the increasing role of digital intermediaries of social interactions, which forms a new context for the problem of justice, opening up perspectives for issues of distance with digital technologies and issues of digital ecology.

Key words: mobility, mobility capital, social order, emergency, justice, COVID-19, pandemic, new normality.

Citation. Karchagin E.V. Justice in the COVID-19 Pandemic: Mobility Capital and New Social Order. Logos et Praxis, 2021, vol. 20, no. 2, pp. 31-38. (in Russian). DOI: https://doi.org/10.15688/lp.jvolsu.2021.2.4
\end{abstract}

УДК 177.9

ББК 87.713 .12

\section{СПРАВЕДЛИВОСТЬ В УСЛОВИЯХ ПАНДЕМИИ COVID-19: МОБИЛЬНОСТНЫЙ КАПИТАЛ И НОВЫЙ СОЦИАЛЬНЫЙ ПОРЯДОК}

\author{
Евгений Владимирович Карчагин
}

Волгоградский государственный технический университет, г. Волгоград, Российская Федерация

\begin{abstract}
Аннотация. Пандемия COVID-19 заставляет пересмотреть концептуальные границы мирового и повседневного социального порядка, затронув такие пары понятий как: естественное / искусственное; привычное / чрезвычайное. Данная статья затрагивает один из аспектов произошедших изменений: трансформацию пространственной мобильности, который, однако, имеет выход и на уровень анализа более глубоких общественных изменений. В первой части опыт изоляции осмысляется на основе теоретических ресурсов социальной теории мобильностей, прежде всего понятий мобильностного капитала и мобильностной справедливости (Дж. Урри, Т. Крессвел, В. Кауфман, М. Шеллер, Н. Кук, Д. Бугц). Не все социальные группы оказались одинаково (им)мобильными, поскольку обладали разным мобильностным капиталом. Проблема мобильностной справедливости приобрела новый контекст - естественной глобальной угрозы, из-за которой усугубились существующие неравенства, обусловленные чрезвычайной ситуацией. Во второй части статьи затрагивается понятие «чрезвычайное положение» Дж. Агамбена и анализируется вопрос трансгрессии системы () мирового социального порядка, включая его повседневное измерение. Ответ на этот вопрос дается на осно-
\end{abstract}




\section{ИЗОЛЯЦИЯ В ОТКРЫТОМ ОБЩЕСТВЕ}

вании анализа интерпретаций и прогнозов ведущих современных европейских интеллектуалов (Дж. Агамбен, С. Жижек, Б. Латур, П. Слотердайк, С. Фуллер). Рассматриваются проблемы социального дистанцирования, трансформация высшего образования, увеличение полномочий государства, сопряженные с медицинскими обоснованиями. Кроме того, важным параметром нового социального порядка является экологический фактор и необходимость социально-критической оптики для осмысления последствий пандемии. Делается прогноз, который фиксирует возрастающую роль цифровых посредников социальных взаимодействий, что формирует новый контекст для проблемы справедливости, открывающий перспективы для вопросов политической дистанции с цифровыми технологиями и цифровой экологии.

Ключевые слова: мобильность, мобильностный капитал, социальный порядок, чрезвычайное, справедливость, COVID-19, пандемия, новая нормальность.

Цитирование. Карчагин Е. В. Справедливость в условиях пандемии COVID-19: мобильностный капитал и новый социальный порядок // Logos et Praxis. - 2021. - T. 20, № 2. - C. 31-38. - DOI: https://doi.org/10.15688/ lp.jvolsu.2021.2.4

\section{Введение}

30 января 2020 г. Всемирная организация здравоохранения объявила вспышку инфекции, вызванную коронавирусом SARS-CoV-2, чрезвычайной ситуацией в области общественного здравоохранения, имеющей международное значение. Длительность и масштаб распространения пандемии COVID-19 в 2020-2021 гг. и ее всестороннего воздействия на сложившийся порядок заставляет поставить вопрос о трансгрессивных эффектах пандемии. При этом важен и глобальный уровень, и уровень повседневности ${ }^{1}$. Поскольку они взаимосвязаны, трудно сказать, где происходят самые серьезные изменения. Однако многие согласятся, что COVID-19 представляет собой «патологический эксперимент на природе наших общественных отношений» [Shapin web]. Действительно, пандемия заставляет пересмотреть концептуальные границы мирового и повседневного социального порядка, затронув такие пары понятий, как естественное / искусственное; привычное / чрезвычайное.

В данной статье затрагивается один из аспектов произошедших изменений - спад, «кризис» пространственной мобильности. В ситуации, вызванной решениями правительств, одни пространства и маршруты перемещений, оказались недоступны, другие - ограниченно доступны. В этой связи в статье я касаюсь: 1) последствий пандемии для социальной жизни, способствовавших углублению существующих неравенств и несправедливости в сфере мобильности; 2) трендов в изменениях социального порядка, определяющих будущее социальности.

\section{Мобильностный капитал}

\section{и справедливость в условиях пандемии}

Изоляция - это феномен, схватывающий сущность «пандемического» опыта. В изоляции люди лишились свободы передвижения, то есть оказались в ситуации, в которой невозможно совершать те движения и перемещения, которые были привычны и доступны ранее, и этот опыт обездвижения требует осмысления.

Для прояснения этого опыта я обращаюсь к социальной теории мобильностей [Урри 2012]. Почему мобильностная парадигма выбрана для осмысления социальных проблем, вызванных пандемией? Основной ее потенциал - теоретическая фундаментальность, то есть это по большому счету теория общества вообще: одновременно и полноценная социальная теория, и философская антропология. Мобильность представляет собой ключ к пониманию того, что такое человек и общество вообще, поскольку люди и социальные группы производят различные виды движения.

Главным ресурсом этой теории для целей статьи я считаю два различения. Первое проводит границу между обычным движением (movement), то есть простым перемещением и мобильностью - перемещением, сопряженным с определенным смыслом. Если движение означает простой факт перемещения из пункта А в пункт Б, то мобильность обязательно связана со значением и смыслом [Cresswell 2006, 9]. Второе различение касается мобильности, то есть социокультурного движения, и «мотильности» (потенциальной мобильности) [Kaufmann, Bergman, Joye 
2004]. В этой связи люди обладают неравным мобильностным капиталом, поскольку мобильность - это ресурс. Не всякий человек обладает одинаковым ресурсом движения. Люди по-разному мобильно «оснащены» и имеют разные возможности совершать нужные для себя перемещения. Не всегда движение есть благо, иногда, когда человеку необходимо остаться на месте и не перемещаться, вынужденное движение представляется как тягота и нежелательное обременение. Соответственно, существуют мобильно бедные и мобильно богатые люди, а также неравномерное распределение мобильности и мобильностного капитала, то есть возможности (и одновременно невозможности) перемещаться или оставаться на месте.

Существенность человеческих передвижений и возможности оставаться на месте выражается в том, что ограничение передвижения и, наоборот, принуждение к перемещениям воспринимаются негативно и являются одной из форм поражения в правах. В изоляции многие люди были вынуждены трансформировать свой привычный режим (им)мобильности.

Карантинные и изоляционные меры в разных странах получили различную степень: если в некоторых регионах Российской Федерации был введен режим чрезвычайной ситуации, то во многих других государствах было объявлено чрезвычайное положение. Многие люди для того, чтобы сохранить возможность обеспечить свои семьи и себя необходимым для жизни, были вынуждены перемещаться по городу, подвергая свою жизнь и жизнь близких риску. У них не было возможности оставаться в совершенной изоляции, в связи с чем обострилось социальное неравенство между теми, кто может выполнять удаленную работу, и теми, кто вынужден совершать поездки каждый день. Пандемия оказалась особенно тяжелым испытанием для жителей индийских трущоб, африканских неформальных поселений и бразильских фавел и в целом для городов Глобального Юга. Однако негативные последствия в отношении риска здоровья испытали и небогатые жители городов Глобального Севера [Cole et al. 2020].

Данные по таким городам, как Барселона и Нью-Йорк, показывают, что пандемия оказала наибольшее влияние на горожан, ко- торые редко занимаются удаленной работой и чьи поездки на работу увеличивают вероятность заражения вирусом [Gutiérrez, Miravet, Domènech 2020]. Иными словами, это общезначимая глобальная проблема.

Социальное положение тех, у кого мобильностный капитал меньше, усугубилось, ухудшилось. В итоге они пострадали больше, нежели те, кто обладали возможностью не совершать этих перемещений или пребывать в своих жилищах, более удобных и менее стесненных для жизни, и имели возможность работать удаленно, то есть владели средствами цифровой коммуникации. В лучшей ситуации оказались люди с более высокими доходами и уровнем жизни, которые не зависят от общественного транспорта, имеют личные автомобили и загородную недвижимость. Это ставит проблему справедливости заново и в новом контексте.

Как таковая проблема справедливости возникает в ситуации необоснованных неравенств, которые могут быть двух типов: естественными и искусственными. С естественным неравенством люди стараются совладать с помощью социокультурных механизмов. Гораздо более негативно воспринимаются неравенства социального или культурного происхождения, у которых нет достаточного обоснования и от этого они вопринимаются как несущественные. Это приводит к возмущению со стороны «чувства» справедливости, что требует соответствующей реакции и понимания. Как пишет Г. Коген, «акцидентальное неравенство несправедливо» [Cohen 2011, VII-VIII].

Следующим инструментом для понимания опыта изоляции я считаю обращение к понятию справедливости, которое неизбежно состоялось и в мобильностной теории, прежде всего благодаря работам американской исследовательницы Мими Шеллер [Sheller 2018]. Действительно, источником той или иной формы порядка мобильности могут быть как естественные причины (климат или стихийные бедствия ${ }^{2}$ ), так и социокультурные факторы, включая действия правительств и различных общественных организаций.

Пандемия оказалась фактором, который превышает силу и масштаб локального бедствия, и «неконкретность», удаленность климатического фактора. Пандемический эффект 
оказался чрезвычайно близким, когда практически все люди на длительное время были лишены возможности совершать привычные передвижения, и наоборот, обычные передвижения стали сопрягаться с опасностью и риском для здоровья и жизни. Пандемию можно назвать естественным фактором чрезвычайного характера. Ограничения для мобильности имелись на всех уровнях - от закрытия государственных и региональных границ до режима индивидуальной самоизоляции, что сделало для большого количества людей близким опыт чрезвычайного. Ранее исследователей справедливости такие факторы привлекали в меньшей степени, однако теперь значимость этого фактора вырастает и чрезвычайный характер этой ситуации тоже требует осмысления. Если привычный опыт жизни располагается на пересечении пары понятий - естественное / природное и социальное / искусственное; то новый опыт оказался периодом продолжительного чрезвычайного, главным фактором которого стал природный феномен вирусной инфекции.

\section{Чрезвычайное положение и «новая нормальность»}

Самым знаменитым теоретиком чрезвычайного является итальянский философ Джорджо Агамбен. Его аналитическая реконструкция истории чрезвычайного положения показала, что европейские власти начиная с римского периода использовали его для отмены или «временной приостановки» права [Агамбен $2011,10]$. В результате с начала ХХ в. чрезвычайное положение становится из исключения правилом, превращаясь в «управленческую технологию» [Агамбен 2011, 16].

Чрезвычайное положение сдвинуло нашу привычную жизнь, ведь это состояние продлилось относительно долго. Природное явление (вирус) было опосредовано государственными властными мероприятиями и постепенно стало частью повседневной рутины. Пандемия как чрезвычайное явление постепенно стало оповседневниваться. Однако любое чрезвычайное положение неизбежно должно смениться нормальным. В этом контексте многих беспокоит вопрос, каким теперь будет социальный порядок, новая «нормальность»?
Будет ли эта ситуация продлеваться дальше и в каком виде? Будет ли откат, и в какой степени, в каких странах и в каком положении кристаллизируется порядок «новой нормальности»? Носит ли трансгрессия временный характер, то есть произойдет ли откат к «доковидным» временам или мы стали свидетелями трансгрессии необратимого характера?

Дж. Агамбен не мог не отреагировать на социально-политические изменения, сопровождавшие пандемию. В своей довольно радикальной книге «Где мы находимся? Эпидемия как политика» [Agamben 2020] он выделил несколько трендов, обозначившихся во время пандемии и имеющих все шансы закрепиться в будущем. Существенными я считаю четыре: трансформация высшего образования; социальное дистанцирование; появление «медицинской религии»; увеличение полномочий государства.

Главнейший из перечисленных трендов сочиальное дистаниирование, опосредованное цифровыми технологиями, на мой взгляд, будет расширяться. Роль цифровых устройств и нечеловеческого фактора при этом многократно возрастет. Тренд на закрытие университетов мне представляется слишком радикально сформулированным. Речь скорее идет о серьезной деформации или трансформации университетской академической жизни, поскольку останется вопрос социализации студентов и профессиональная социализация будущих ученых. Уничтожение университетов не сможет решить задачу воспроизводства кадров высшей квалификации.

Увеличение полномочий государства сопряжено с появлением медицинской религии. Согласно Агамбену, во время пандемии возникла новая управленческая парадигма: «политика биобезопасности». Новое положение вещей таково, что опасность представляет каждый человек. Под подозрением оказывается не условный выходец из арабских стран и носитель стереотипных черт радикального экстремиста, а человек без маски, человек чихающий; каждый человек становится подозрительным и подозреваемым. В связи с этим можно предположить, что мировой порядок претерпит существенные изменения, подобно тому, как это случилось после 11 сентября 2001 г., когда была объявлена война против 
международного терроризма, а принцип безопасности позволил нарушать принципы личной неприкосновенности и иметь правительствам «тоталитарный характер». Можно упомянуть появившуюся после этого «инфраструктуру безопасности», ставшую привычной в транспортных узлах. Возможно, что теперь она «обогатится» медицинской составляющей.

Не все исследователи в своих высказываниях столь же радикальны и критичны, как Агамбен. Б. Латур, например, находит большую пользу в том, что у людей появилось время побыть наедине с собой, поразмышлять. Вместе с этим обнаружилась польза в том, что люди занимались эпистемологическими вопросами, обсуждая статистику и степень доверия данным. Однако самый большой урок заключается в том, чтобы понять, что вирусы внутри нас и нам нужно научиться жить с ними: «Это огромный эксперимент. Это глобальная катастрофа, которая пришла не извне, как война или землетрясение, а изнутри. Вирусы полностью внутри нас. Мы не можем полностью их выбросить. Мы должны научиться жить с ними» [Watts web]. Мы заперты в «критической зоне», мы находимся внутри Геи, Жизни с большой буквы [Watts web]. Л. Брайант присоединяется к Б. Латуру, отмечая важность темы материальности как ответственности перед планетой: «Мы сталкиваемся с планетарным коррелятом, который требует переосмысления нашего отношения к материальности и нашему материальному воплощению» [Bryant web].

Своеобразный синтез акцентов Агамбена и Латура можно найти у Р. Эспосито. В своем критическом развороте он отмечает парадоксальность самого выражения «социальная дистанция»: «Дистанцирование не может быть социальным, и дистанцирование всегда приводит к десоциализации и сокращению коммунальных форм жизни» [Esposito web]. Пандемия в очередной раз продемонстрировала несправедливость и неравенство современного капиталистического мирового порядка, что ставит вопрос о его альтернативах. Р. Эспосито указывает в этой связи на «третий вариант собственности»: «Частные блага принадлежат частным лицам, а общественные блага принадлежат государству, но общее достояние (commons) - это блага, которые принад- лежат всем одинаково, поскольку они не допускают никаких претензий на исключительную собственность» [Esposito web]. В своем экологическом развороте он утверждает, что действенным ответом капитализму может стать экология: «Капиталистическая экономика должна двигаться к модели, которая более совместима с экологическими ценностями. Он должен пройти радикальную экологическую реформу, зеленую реформу. Для некоторых людей оцифровка - это даже частичный ответ» [Esposito web].

Известный словенский философ С. Жижек осмысляет пандемию с левых, критических позиций. С его точки зрения, нас ожидает двойная альтернатива будущего мирового порядка: обновленный коммунизм или новое варварство «с человеческим лицом», проявившееся в пандемию как «безжалостные меры выживания, применяемые с сожалением и даже сочувствием, но легитмированные экспертными мнениями» [Žižek 2020, 86] и как восприятия коронавируса в качестве «полезной инфекции, которая позволяет человечеству избавиться от старых, слабых и больных, подобно тому как вырвать полусгнившие сорняки, чтобы молодые, более здоровые растения могли процветать и таким образом способствует глобальному здоровью» [Žižek 2020, 69]. В этом смысле сама по себе экологическая оптика может иметь «обратное» измерение, негативную сторону. Само по себе принятие в расчет природных явлений и их важности не гарантирует избавления от худших форм евгеники и витализма.

В свою очередь, С. Фуллер ставит серьезный вопрос о реактивной и проактивной стратегиях взаимодействия с пандемией в будущем: «Приверженцы принципа предосторожности склонны предполагать, что человечество «всегда уже» подчинено некоему универсальному внешнему агенту, называемому, скажем, «Природа» или «Гея», в терминах которого мы можем находиться либо в равновесии, либо в конфликте. В любом случае этот оппонент не является предметом переговоров: он устанавливает предел допустимой деятельности. Напротив, приверженцы принципа проактивности склонны предполагать более игровые отношения с оппонентом, при этом заратустрийский императив Ницше «что 
не убивает меня, делает меня сильнее» действительно применяется. Это подходит для мира бесконечных пандемий, в котором противник может находиться на данный момент, но может видоизменяться, чтобы создать угрозу в будущем, что, в свою очередь, может потребовать радикального пересмотра своих modus operandi и даже raison d'être, включая инкорпорацию ранее невидимого «другого»»» [Fuller 2020, 555].

В этом контексте мне кажется более реалистичной «материально-цифровая» версия «новой нормальности». Я считаю, что ее теоретическое описание и осмысление возможно благодаря обращению к материальному повороту в социально-гуманитарном знании. В последние десятилетия планомерно возрастала роль материального и вещного фактора в наших социальных опосредованиях. В частности, цифровые технологии достигли такого уровня развития, что теперь они заявляют о себе и, вероятнее всего, станут полноправными участниками социальной жизни. Пандемия помогла проявиться и укрепиться новым «субъектам» социальной жизни. Эти посредники по всей видимости окажутся «в выигрыше» в этой новой реальности и новой нормальности.

П. Слотердайк говорит о необходимости менять «грамматику нашего поведения» [Sloterdijk web], отмечая готовность большого числа людей соблюдать меры предосторожности и дистанцирование. Пандемия прежде всего показала, что мы живем в одно время: «Человечество достигло ситуации синхронности на основе потока информации. Мы действительно глобально связаны и все больше и больше живем в одном временном измерении» [Sloterdijk web]. Относительно успешное поддержание дистанции, синхронизация коммуникаций в большой степени стали возможными именно благодаря уровню развития и повсеместности цифровых технологий. Проблема справедливости нового порядка тем самым циифровизируется.

\section{Заключение}

Неравномерный мобильностный капитал в сочетании с экономическим «прототипом» сказался на возможностях людей трансфор- мировать привычные практики (им)мобильности. Перемещения и воздержание от них оказались сопряжены с угрозой здоровью и жизни не от вируса, а от социально-экономических причин. В период изоляции не все люди оказались готовыми к «новой нормальности», вызванной пандемией.

Обретем ли мы новый мировой порядок, более несправедливый, чем прежде, или несправедливый по-другому? Судя по всему, меняется контекст проблемы справедливости. В структуры и порядки мобильного неравенства и несправедливости включены не только люди, но и разнообразные нечеловеческие факторы. Общественная реальность в больше степени будет пронизана цифровыми технологиями. В связи с этим можно предположить снижение в будущем пространственной мобильности. Поскольку серьезный урон претерпел туризм, то есть основания ожидать развитие дигитального туризма.

Другой важный момент касается улучшения в довольно короткий срок экологической ситуации на планете, в результате чего природа и «естественное» оказались в выигрыше. В связи с этим, возможно, следует ожидать возрастание экологического фактора в общественной жизни, и практиках и инфраструктуре мобильностей. Весьма вероятно увеличение доли экологически безопасных видов транспорта.

Вирус во многом выступил как концептуальное орудие, объединяющее людей, схватывающее человечество как единство на новых основаниях. Во время пандемии снова был поставлен вечный вопрос: «что значит быть человеком?» Определяется ли он через движение, прикосновение, использование цифровых посредников и т. д.? Возможно, нам стоит обдумать вопрос о «политической» дистанции с цифровыми технологиями, а не с людьми. Возможно, существенную роль в общественных и теоретических дискуссиях будут играть вопросы цифровой экологии.

\section{ПРИМЕЧАНИЯ}

\footnotetext{
${ }^{1}$ См. анализ городской повседневности в условиях пандемии: [Карчагин, Назарова, Янин 2020].

${ }^{2}$ Канадские исследователи Н. Кук и Д. Бутц так описывают последствия стихийного бедствия в
} 
северной части Пакистана: «в контексте оползневой катастрофы в Аттабаде, мобильностная справедливость повлечет за собой демократическое государственные процедуры принятия решений, способствующие участие Годжалиса в определении коллективной стратегии для борьбы с суженной мобильностью, средства для разработки адаптированных способностей к мобильности на индивидуальном уровне, и условия, которые позволяют эти возможности реализовать [Cook, Butz 2016, 5].

\section{СПИСОК ЛИТЕРАТУРЫ}

Агамбен 2011 - Агамбен Дж. Ноmo sacer. Чрезвычайное положение. М.: Издательство «Европа», 2011.

Карчагин, Назарова, Янин 2020 - Карчагин E.B., Назарова М.П., Янин К.Д. Городская повседневность в условиях пандемии COVID-19 // Социология города. 2020. № 4. С. 42-50.

Урри 2012 - Урри Дж. Мобильности. М.: Праксис, 2012.

Agamben 2020 - Agamben G. A che punto siamo? L'epidemia come politica. Macerata: Quodlibet, 2020.

Bryant web-Bryant L.R. A World Is Ending [Identities: Journal for Politics, Gender and Culture]// https:// identitiesjournal.edu.mk/index.php/IJPGC/ announcement/view/21.

Cohen 2011 - Cohen G.A. On the Currency of Egalitarian Justice, and Other Essays in Political Philosophy. Princeton; Oxford: Princeton University Press, 2011.

Cole et al. 2020 - Cole H., Anguelovski I., Baro F., Lamarca M.G. TheCOVID-19 Pandemic: Power and Privilege, Gentrification, and Urban Environmental Justice in the Global North // Cities \& Health. 2020. P. 1-5.DOI: 10.1080/23748834.2020.1785176.

Cook, Butz 2016-Cook N., Butz D. Mobility Justice in the Context of Disaster // Mobilities. 2016. Vol. 11. № 3. P. 400-419.

Cresswell 2006 - Cresswell T. On the Move: Mobility in the Modern Western World. N. Y.: Taylor \& Francis, 2006.

Esposito web - Esposito R. The Biopolitics of Immunity in Times of COVID-19: An Interview with Roberto Esposito [Antipode Online] // https://antipodeonline.org/2020/06/16/ interview-with-roberto-esposito/.

Fuller 2020 - Fuller S. A Post-Truth Proactionary Look at the Pandemic // Postdigital Science and Education. 2020. Vol. 2, № 3. P. 551-555.

Gutiérrez, Miravet, Domènech 2020 - Gutiérrez A., Miravet D., Domènech A. COVID-19 and Urban Public Transport Services: Emerging Challenges and Research Agenda // Cities \& Health. 2020. P. 1-4. DOI: 10.1080/23748834.2020.1804291. Kaufmann, Bergman, Joye 2004 - Kaufmann V., Bergman M.M., Joye D. Motility: Mobility As Capital // International Journal of Urban and Regional Research. 2004. Vol. 28, №4. P. 745-756.

Shapin web - Shapin S. COVID and Community // https://lareviewofbooks.org/article/covidand-community/.

Sheller 2018 - Sheller M. Mobility Justice: The Politics of Movement in an Age of Extremes. L.; N. Y.: Verso, 2018.

Sloterdijk web - Sloterdijk P. Humans are Not Prepared to Protect Nature // https://www.dw.com/en/howdo-we-change-peter-sloterdijk-environmentcoronavirus-on-the-green-fence-climatechange/a-53533840.

Žižek 2020-Žižek S. Pandemic!: Covid-19 shakes the world. N. Y.; L.: OR Books, 2020.

Watts web-Watts J. Bruno Latour: "This is a Global Catastrophe That Has Come from Within" [The Guardian] // https://www.theguardian.com/ world/2020/jun/06/bruno-latour-coronavirusgaia-hypothesis-climate-crisis.

\section{REFERENCES}

Agamben G., 2011. Homo Sacer. State of Emergency. Moscow, Europe Publ.

Karchagin E.V., Nazarova M. P., Yanin K. D. 2020. Urban Daily Life in the COVID-19 Pandemic. Sotsiologiya Goroda, no. 4, pp. 42-50.

Urri Dzh., 2012. Mobilities. Moscow, Praksis.

Agamben G., 2020. A che punto siamo? L'epidemia come politica. Macerata, Quodlibet.

Bryant L.R., 2020. A World Is Ending. Identities: Journal for Politics, Gender and Culture. URL: https://identitiesjournal.edu.mk/index.php/ IJPGC/announcement/view/21.

Cohen G.A., 2011. On the Currency of Egalitarian Justice, and Other Essays in Political Philosophy. Princeton; Oxford, Princeton University Press.

Cole H. et al., 2020. The COVID-19 Pandemic: Power and Privilege, Gentrification, and Urban Environmental Justice in the Global North. Cities \& Health, 2020, pp. 1-5. DOI: 10.1080/23748834.2020.1785176.

Cook N., Butz D., 2016. Mobility Justice in the Context of Disaster. Mobilities, vol. 11, no. 3, pp. 400419. DOI:10.1080/17450101.2015.1047613.

Cresswell T., 2006. On the Move: Mobility in the Modern Western World. New York, Taylor \& Francis.

Esposito R., 2020. The Biopolitics of Immunity in Times of COVID-19: An Interview with Roberto 
Esposito [Antipode Online]. URL: https:// antipodeonline.org/2020/06/16/interview-withroberto-esposito/.

Fuller S., 2020. A Post-Truth Proactionary Look at the Pandemic. Postdigital Science and Education, vol. 2, no. 3, pp. 551-555.

Gutiérrez A., Miravet D., Domènech A., 2020. COVID-19 and Urban Public Transport Services: Emerging Challenges and Research Agenda. Cities \& Health, pp. 1-. DOI:10.1080/23748834.2020.1804291.

Kaufmann V., Bergman M.M., Joye D., 2004. Motility: Mobility as Capital. International Journal of Urban and Regional Research, vol. 28, no. 4, pp. 745-756.

Shapin S., 2020. COVID and Community. URL: https:// lareviewofbooks.org/article/covid-and-community.
Sheller M., 2018. Mobility Justice: The Politics of Movement in an Age of Extremes. London; New York, Verso.

Sloterdijk P., 2020. Humans are Not Prepared to Protect Nature. URL: https://www.dw.com/en/how-dowe-change-peter-sloterdijk-environmentcoronavirus-on-the-green-fence-climatechange/a-53533840.

Žižek S., 2020. Pandemic!: Covid-19 Shakes the World. New York, London, OR Books.

Watts J., 2020. Bruno Latour: "This is a Global Catastrophe That Has Come from Within". The Guardian. URL: https://www.theguardian.com/ world/2020/jun/06/bruno-latour-coronavirusgaia-hypothesis-climate-crisis.

\section{Information About the Author}

Evgeniy V. Karchagin, Doctor of Sciences (Philosophy), Associated Professor, Department of Philosophy, Sociology and Psychology, Volgograd State Technical University, Akademicheskaya St, 1, 400074 Volgograd, Russian Federation, evgenkar@yandex.ru, https://orcid.org/0000-0001-7398-9292

\section{Информация об авторе}

Евгений Владимирович Карчагин, доктор философских наук, профессор кафедры философии, социологии и психологии, Волгоградский государственный технический университет, ул. Академическая, 1, 400074 г. Волгоград, Российская Федерация, evgenkar@yandex.ru, https://orcid.org/0000-0001-7398-9292 